\title{
How are so many foreign arboviruses introduced in Brazil?
}

\author{
Luiz Tadeu Moraes Figueiredo ${ }^{[1]}$
}

[1]. Centro de Pesquisa em Virologia, Faculdade de Medicina de Ribeirão Preto, Universidade de São Paulo, Ribeirão Preto, São Paulo, Brasil.

To be maintained in nature, arboviruses (arthropod-borneviruses) go through complex cycles involving vertebrate hosts and hematophagous vectors (mosquitoes, ticks, sandflies) that carry and transmit the virus to other organisms. The host-vector conjunction, represented by two completely different animals, depends on the congruence of biology, geography, climate, ecology, and even economic factors. Although apparently improbable, this congruence of factors occurs, accounting for the existence of so many arboviruses, including some that produce human disease. More surprising is the rapid emergence of an arbovirus introduced in a new geographical area, after establishing new maintenance cycles based on the complex host-vector conjunction, as with some arboviruses introduced from distant global regions to the tropical Americas. Among these emerging arboviruses, we highlight those related with the mosquito Aedes aegypti; this vector has affinity for human blood and lives in houses, facilitating urban cycles where man, instead of other animals, becomes a viral reservoir, facilitating population-wide pathogen spread and large epidemics. This happens with yellow fever (in the urban cycle), dengue virus (DENV), chikungunya virus (CHIKV), and Zika virus (ZIKV).

Interestingly, the introduction of new arboviruses in Brazil has been reported since the $19^{\text {th }}$ century. In December 1848 , yellow fever virus (Flaviviridae, Flavivirus), an African arbovirus, was introduced in Rio de Janeiro City by sailors of the North American ship Navarre. Some of these men lodged downtown, on Misericordia Street. A Swedish sailor became icteric, vomited a dark fluid, and died the next day. Then, other sailors became sick, signifying the beginning of a horrible sequence of outbreaks. In 1850, the Central Commission of Public Health of Rio de Janeiro explanation for the causes of the yellow fever epidemic was very discouraging: Indigestion, the suspension of perspiration, exposure to rain, night humidity and burning sunshine were without a doubt one of the strongest causes of the disease among us. The fatigues of the spirit, the

Corresponding author: Dr. Luiz Tadeu Moraes Figueiredo.

e-mail:1tmfigue@fmrp.usp.br

Received 25 November 2016

Accepted 28 November 2016 moral contraries, the violent passions, the terror, etc., also contribute much to its development. Therefore, action was not possible; between 1850 and 1902, yellow fever occurred annually in Rio de Janeiro, with the exception of only 3 years (1865, 1866, and 1867), resulting in an impressive 58,063 deaths. The disease was only eradicated after it became known that the virus is transmitted by Aedes aegypti. Yellow fever was eradicated in Rio de Janeiro in 1909, after Oswaldo Cruz's 6-year battle against the mosquito ${ }^{(1)}$.

The discovery of the yellow fever 17D vaccine in 1937 and subsequent large-scale vaccination ceased the urban transmission of yellow fever ${ }^{(1)}$. However, this virus is currently maintained in sylvatic epizootics involving primates and eventually humans and is a permanent threat with urbanization that requires both permanent attention and continuous vaccination.

All four serotypes of dengue virus (Flaviviridae, Flavivirus) are originally zoonotic of primates from southeast Asia. A dengue virus produced outbreaks in Rio de Janeiro and Niterói City in the 1920s. At this time, Antonio Pedro produced a fantastic clinical description of classic dengue based on his patients ${ }^{(2)}$. Then, dengue disease was not reported in Brazil for more than 50 years. However, these viruses have been serially reintroduced since the 1980s; in March 1986, dengue virus serotype 1 (DENV-1) of the American genotype V started an outbreak in Nova Iguaçu City, in the metropolitan area of Rio de Janeiro. The movement of people facilitated the rapid spread of DENV-1 among the completely susceptible population with serological surveys estimating $3,000,000$ dengue cases $^{(3)}$. DENV-1 was probably introduced from the Caribbean or Northern countries of South America. However, details on its occurrence are lacking.

Dengue virus serotype 2 (DENV-2) genotype III (Jamaica) emerged in Brazil, causing a major epidemic in Rio de Janeiro in 1990. Ten years later, the same occurred with dengue virus serotype 3 (DENV-3) of genotype III (Indian subcontinent). In both cases, the viruses were introduced more than once, from the Caribbean or from Northern South American countries.

This reintroduction was clearly demonstrated for DENV-3 in North Brazil, after the first outbreak in Rio de Janeiro. As with DENV-1 and DENV-2, DENV-3 spread rapidly throughout the country. The co-circulation of these different dengue serotypes 
resulted in a large number of severe cases and fatalities. In fact, until 2009, DENV were responsible for approximately 5 million cases, 15,000 cases of hemorrhagic fever, and about 1,000 deaths ${ }^{(3)(4)}$.

In an interesting case, dengue virus serotype 4 (DENV-4) of genotype II was introduced in Roraima in 2010, probably from the Caribbean islands, Venezuela, or Colombia. This same state previously experienced a restricted DENV-4 outbreak in 1981. In 2010, the virus produced large outbreaks throughout Brazil $^{(4)}$. However, a distinct DENV-4, belonging to genotype I, that was never reported in the Americas has infected patients from Manaus City, in the Amazon State, since 2005. This rather unexpected virus was likely introduced directly to Manaus by intense economic activity and movement of people from Asian companies, particularly from China ${ }^{(5)}$. Years later, the same DENV-4 of genotype I was reported in Northeast Brazil.

All dengue serotypes were successfully introduced in Brazil during the last 35 years and have remained in the country producing a large number of outbreaks transmitted by Aedes aegypti, leading to $>10,000,000$ people with disease and thousands of deaths ${ }^{(4)}$. In 2016, the first available tetravalent anti-dengue vaccine, developed by Sanofi Pasteur, was administered as a 3-dose series on a 0/6/12-month schedule. Although this vaccine is not highly immunogenic against the four viral serotypes, it has an $80 \%$ efficacy against severe dengue, recommending its use.

Chikungunya virus (CHIKV, Togaviridae, Alphavirus), originally an arbovirus of African primates, was introduced to the Americas from Asia, through the Caribbean, and the first cases, transmitted by Aedes aegypti and probably by Aedes albopictus, were reported in December 2013. In March 2014, this Asian genotype virus produced $>15,000$ cases of febrile illness with joint pain. In August 2014, cases were reported in Venezuela and Colombia. In September 2014, the first CHIKV outbreak in Brazil occurred in the Northern State of Amapá, and an outbreak by another genotype of CHIKV (ECSA genotype), probably introduced from Angola in 2013 or 2014, occurred in the Northeastern State of Bahia. Thousands of cases were reported, and the virus spread rapidly through the completely susceptible population of Brazil. In addition, CHIKV was added to Mayaro virus (MAYV), an American arbovirus of primates belonging to the same genera, that produces small outbreaks and sporadic cases of febrile illness with joint pain in the North and Midwest of the country. Thus, the differential diagnosis between CHIKV and MAYV is important in these regions ${ }^{(6)}$. Until September 2016, >236,000 cases of CHIKV were reported including a large number of patients that evolved with chronic arthropathy ${ }^{(4)}$. Moreover, 120 CHIKV-related deaths were reported in November, mostly in the elderly and people with comorbidities. At least 2 CHIKV vaccines are in phase 2 evaluation, one containing virus-like particles and another vectored by the measles virus; they might become available in a couple of years, allowing the control of this virus.

The last arbovirus introduced in Brazil was Zika (ZIKV, Flaviviridae, Flavivirus), also a zoonotic virus of African primates. This virus considerably worsened the epidemiological scenario involving arboviruses. ZIKV of Asian lineage was probably introduced in 2013 or 2014 by travelers from the Western Pacific Islands and was first reported in February 2015 in Bahia State. The virus quickly spread to other Northeastern states and subsequently other regions of the country. Known as being causative of benign acute febrile illness accompanied with rash and conjunctivitis, the virus was not initially considered a relevant problem. However, its importance as a public health problem has increased substantially since ZIKV was associated with surprisingly severe diseases in Brazil. First, cases of flaccid paralysis from Guillain-Barré syndrome were associated with the viral infection. More importantly, in September 2015, cases of severe ZIKV-related congenital disease that produced extensive destruction of the central nervous system were associated with maternal infection ${ }^{(7)}$. As of August 2016, 196,976 probable cases of ZIKV in the entire country were reported. In addition, 16,264 suspected cases were reported in pregnant women, and $>3,000$ babies had microcephaly related with congenital ZIKV infection ${ }^{(4)}$.

Outbreaks and scientific studies indicate that this neurotropic virus has a particular affinity for neural progenitor cells leading to extensive destruction of the central nervous system during embryogenesis. This dramatic outcome of children with microcephaly, a severe and irrecoverable neurological disease, has shocked the world; as in developed countries, Brazil should legally offer abortions of these fetuses with confirmed disease. Newborns presenting with less severe manifestations of ZIKV congenital disease are beginning to be reported. ZIKV has also produced meningoencephalitis in people of all ages, including immune-depressed patients. Vaccines are promising for the control of future ZIKV outbreaks. A deoxyribonucleic acid (DNA) vaccine is being tested, and other vaccines are also in preparation.

Brazil seems to be a suitable destination for the introduction of arboviruses with new features. CHIKV and ZIKV have strange characteristics that are uncommon in arboviral infections, including congenital disease and chronic maintenance of the pathogen. We now know that CHIKV and ZIKV find refuge in organs that protect against aggression by the immune system, such as CHIKV in bone tissue, where it produces an unpublished viral osteomyelitis. ZIKV can be detected for long periods in the placenta after an acute maternal infection, central nervous systems of the fetus and newborn, and the testes after infection, the latter allowing sexual transmission.

\section{Conflicts of interest}

The author declares that there is no conflict of interest.

\section{REFERENCES}

1. Franco O. História da febre amarela no Brasil. Rio de Janeiro: Ministério da Saúde; 1969. 208p.

2. Pimentel AP. O dengue em Nictheroy. Brazil-Médico1923; 1:174-177.

3. Figueiredo LTM. Dengue in Brazil during 1999-2009: a review. Dengue Bull 2010; 34:6-12.

4. Ministério da Saúde. Secretaria de Vigilância em Saúde. Dengue, 
Chikungunya e Zika: 2000 a 2010. Informes epidemiológicos. Brasília: Ministério da Saúde; 2016.

5. de Melo FL, Romano CM, de Andrade Zanotto PM. Introduction of dengue virus 4 (DENV-4) genotype I into Brazil from Asia? PLoS Negl Trop Dis 2009, 3:e390. doi: 10.1371/journal.pntd.0000390.
6. Figueiredo MLG, Figueiredo LTM. Emerging alphaviruses in the Americas: Chikungunya and Mayaro. Rev Soc Bras Med Trop 2014; 47:677-683.

7. Figueiredo LTM. The recent arbovirus disease epidemic in Brazil Rev Soc Bras Med Trop 2015; 48:233-234. 